\title{
Tutorial: Ultra-high Spatial Resolution EBSD: Transmission Kikuchi Diffraction (TKD) in the SEM
}

\author{
Scott Sitzman ${ }^{1}$ \\ 1. The Aerospace Corporation, Physical Sciences Laboratory, E1 Segundo, USA.
}

EBSD analysts who may encounter microstructures with grain sizes on the order of $100 \mathrm{~nm}$ or less will benefit from an understanding of Transmission Kikuchi Diffraction (TKD) and its requirements and best practices, especially since all modern, conventional EBSD systems on FEG-SEMs are readily capable of the technique. TKD involves the analysis of Kikuchi patterns in transmission from thin samples rather than in reflection from bulk and consequently brings significant spatial resolution advantages [1], but is otherwise almost identical to the established EBSD technique in most other ways (e.g., the physics of pattern generation, automated pattern capture \& indexing, and the post-collection analysis of resulting data). Nevertheless, since TKD is typically applied to challenging samples certain considerations become paramount for success. This tutorial focuses techniques and strategies to help existing EBSD users have confidence in their ability to apply TKD when it best suits their analytical goals.

TKD's superior spatial resolution arises from the use of near incident energy electrons which carry diffraction information from very near the exiting surface (good depth resolution) and from a beam which has not experienced a lot of scatter / beam broadening as it traverses the thin sample (good lateral resolution). Sample section thickness, material density and SEM conditions affect both of these [2]. Extremely fine microstructures $(\sim 50 \mathrm{~nm}$ or lower grain size), especially in higher density materials, require thinner (e.g., under $100 \mathrm{~nm}$ ) sample sections to minimize lateral scatter; higher accelerating voltage (30 kV is typical) also helps slightly but increases elastic \& inelastic mean free path lengths and worsens depth resolution. Poor depth and/or lateral resolution with respect to the fineness of the microstructure can result in overlapped patterns and degraded indexing. Modern EBSD indexing algorithms are good at solving even with some degree of pattern overlap, but minimizing this situation should be a primary goal of the user.

Collection speed is a very important consideration due to drift, which is always a factor at the mapping resolutions where TKD becomes preferred over EBSD, and so the analyst must use collection speeds that minimize its impact and/or use software drift correction tools while maintaining high data quality. Higher collection speed also reduces contamination, which can become pronounced and decrease diffraction pattern quality as mapping progresses, particularly at smaller step sizes. Pre-mapping considerations are very important for both issues, including allowing the SEM vacuum level to stabilize and the stage to completely settle prior to mapping. Sample/chamber cleanliness and plasma cleaning are both useful in mitigating contamination.

Another acquisition setting involving an important tradeoff is sample back-tilt [3]. Higher quality patterns are collected with side-entry (standard) EBSD detectors when samples are tilted away from them, but increasing back-tilt reduces spatial resolution since the intersection of the exit surface with the broadening beam becomes larger. This effect becomes pronounced at a few tens of degrees, but in practice $20-30^{\circ}$ of back tilt is a good compromise. TKD-customized detectors with phosphor screens placed directly under the sample removes the necessity of back-tilting. 
Diode-based forescatter electron detectors and EBSD detector phosphor screen-based analogues (both referred to here as "FSDs") are very useful imaging tools for TKD, since standard backscatter electron detectors positioned above horizontal or near-horizontal thin samples are not useful for orientation contrast imaging. FSDs, depending on positioning with respect to the primary beam, act as ADF- or HAADF-STEM detectors, or even BF-STEM if placed directly under the primary beam. The higherangle geometries are more typical and carry some $\mathrm{Z}$ information at the expense of diffraction (orientation) information, but special settings can mitigate this effect: Combined use of two (or more) diodes / capture regions on the phosphor screen with symmetrical geometries with respect to the sample tilt direction, where the contrast from one of the two is inverted. Combining the signals effectively negates $\mathrm{Z}$-contrast and strengthens orientation contrast.

As in EBSD, the simultaneous collection of EDS information is often useful in TKD but the differences in origination volumes is even more pronounced. Diffraction information in the Kikuchi pattern is dominated by near-exit (bottom) surface scattering, whereas x-rays generated throughout the thin section are almost equally able to escape the sample towards the EDS detector. This must be borne in mind for proper TKD-EDS data comparison and interpretation. X-ray generating interaction volumes in TKD conditions (thin samples at high $\mathrm{kV}$ ) are smaller than in typical EBSD conditions, so a resolution improvement is realized for EDS information as well.

\section{References:}

[1] R. Keller and R. Geiss, Journal of Microscopy 245 (2012), p. 245.

[2] P. Trimby, Ultramicroscopy 120 (2012), p. 16.

[3] G. Sneddon, P. Trimby and J. Cairney, Microscopy and Microanalysis 23 (2017), p. 532.
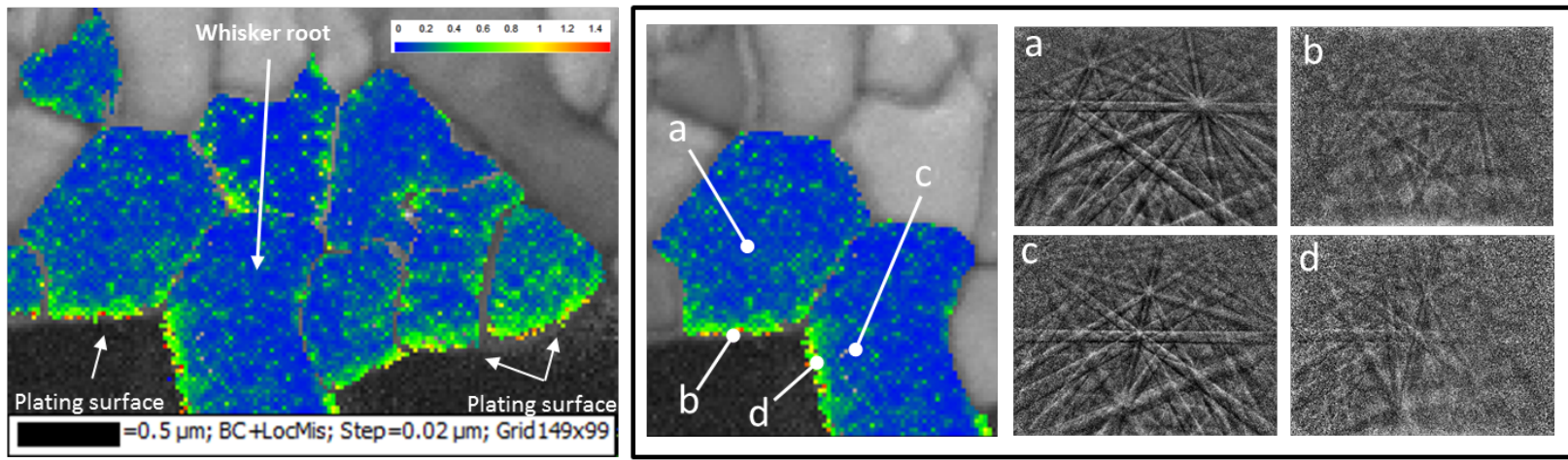

Figure 1. TKD Kernel Average Misorientation (KAM)-type plastic strain map of a whisker root (elongated grain extending to map bottom) and surrounding grains in a tin plating, with patterns shown at selected points. Pixels are colored by an average of the measured change in crystallographic orientation with respect to each neighboring pixel; coloring toward the red end of the rainbow scale indicate greater degrees of mis-orientation. This map demonstrates the need for special care and consideration in recognizing artifacts and interpreting data in TKD. (Left) KAM map showing apparently higher strain at the plating surface and whisker edge. (Right) Patterns at these positions (b and d, respectively) show anomalous thickness and edge effects, increasing orientation measurement error and giving unreliable KAM values. 